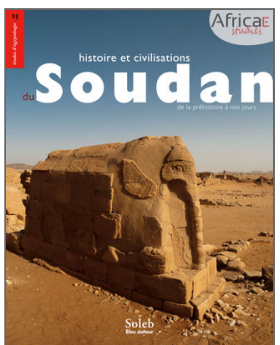

Olivier Cabon (dir.)

Histoire et civilisation du Soudan

De la préhistoire à nos jours

\title{
11. La victoire de l'islam
}

Ottomans et Fungs. 1500-1820

\section{Claude Rilly}

DOI : 10.4000/books.africae. 2837

Éditeur : Africae, Soleb, Bleu autour

Lieu d'édition : Paris, Khartoum

Année d'édition : 2017

Date de mise en ligne : 17 janvier 2022

Collection : Africae Studies

EAN électronique : 9782493207074

\section{Q OpenEdition}

\section{Books}

http://books.openedition.org

\section{Référence électronique}

RILLY, Claude. 17. La victoire de l'islam : Ottomans et Fungs. 1500-1820 In : Histoire et civilisation du Soudan : De la préhistoire à nos jours [en ligne]. Paris, Khartoum : Africae, 2017 (généré le 28 janvier 2022). Disponible sur Internet : <http://books.openedition.org/africae/2837>. ISBN : 9782493207074. DOI : https://doi.org/10.4000/books.africae.2837. 


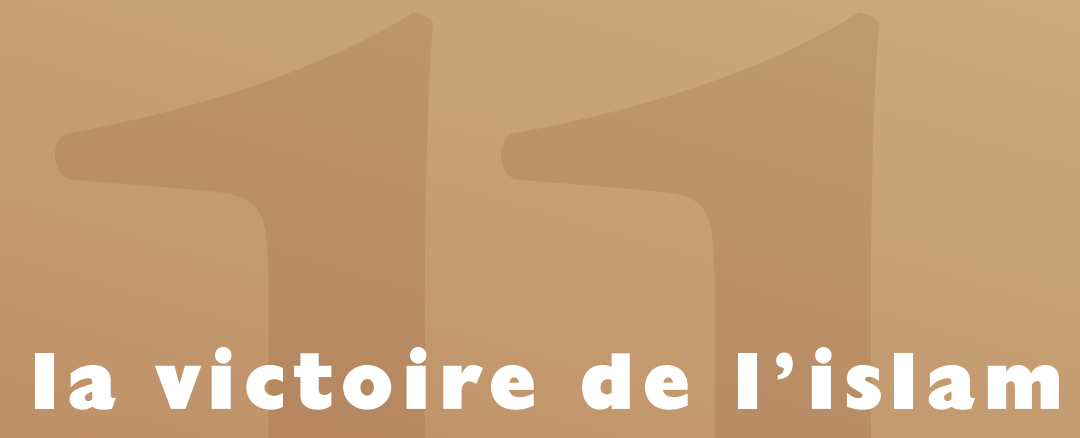

Ottomans et Fungs

$1500-1820$ 
Lorsque les premiers voyageurs occidentaux pénétrèrent au Soudan, à la fin du XVIII ${ }^{e}$ siècle, ils ne rencontrèrent plus aucune trace vivante du christianisme. Les coptes, assez nombreux, qui résident aujourd'hui au Soudan et possèdent leurs églises, descendent tous d'Égyptiens immigrés et ne constituent donc pas une survivance de la chrétienté nubienne. En moins de trois siècles, la foi chrétienne a été complètement supplantée par l'islam. Cette disparition rapide s'explique par la situation géographique et politique du pays. Après la chute du Dotawo et d'Alodia, au début du $\mathrm{xvI}^{\mathrm{e}}$ siècle, il n'existait plus d'État chrétien au Soudan, alors que l'Abyssinie avait pu préserver sur les hauts plateaux un royaume orthodoxe monophysite, pourtant cerné de principautés musulmanes. Mais il n'existait pas davantage d'État musulman puissant et centralisé qui, à l'instar de l'Égypte médiévale, eût pu s'accommoder de la présence en son sein de communautés chrétiennes et, moyennant un impôt spécifique, leur assurer une protection, notamment contre les tribus arabes esclavagistes qui menaçaient les populations non musulmanes. Enfin, l'isolement de la Nubie, renforcé par l'insécurité des routes vers l'Égypte, empêchait le renouvellement du clergé. Une ambassade portugaise présente en Éthiopie dans les années 1520 à 1527 relate qu'une délégation de chrétiens nubiens était venue demander au monarque abyssin de leur envoyer des prêtres, ce que le roi déclina, disant qu'il avait lui-même le plus grand mal à obtenir du patriarche d'Alexandrie l'envoi d'un métropolite.

\section{Les Ottomans dans le nord de la Nubie}

En 1517, le sultan ottoman Sélim Ier conquit l'Égypte, mettant fin au pouvoir suprême des Mamelouks. Il en maintint certains comme gouverneurs de provinces et administrateurs, tout en les plaçant sous le strict contrôle de la Sublime Porte. La mainmise du nouveau pouvoir sur l'ensemble du territoire fut toutefois une œuvre de longue haleine, car la Haute-Égypte était tenue par des tribus arabes qu'il fallut réduire une par une. Dans les dernières années du règne suivant, celui de Soliman le Magnifique (15201566), l'ensemble du pays était enfin aux mains des Ottomans. Le sultan envisagea alors d'étendre son Empire en Afrique et, ce faisant, de protéger les frontières égyptiennes, et il dépêcha à cet effet le général Özdémir Pacha. Celui-ci partit avec une armée de 40000 hommes vers le Soudan, où il s'empara de Qasr Ibrim.

Il laissa en Nubie plusieurs bataillons bosniaques avec la mission de pacifier le territoire et de construire des forteresses, dont les principales furentbâtiesà Assouan, à Qasr Ibrim et dansl'île de Saï, c'est-à-diresur les lieux stratégiques qui permettaient le contrôle de la Basse et de la Moyenne-Nubie. 
Özdémir Pacha continua sa mission en s'emparant de vastes portions de l'Abyssinie et de la côte de la mer Rouge, notamment la cité de Massaoua, aujourd'hui en Érythrée.

Afin d'administrer la Nubie et de faire rentrer l'impôt, il installa des potentats locaux, appelés "kachefs", qui jusqu'à la conquête de Méhémet Ali, en 1820, devaient rester les seules autorités du nord du Soudan. Ces kachefs étaient originellement des officiers ottomans, souvent des Mamelouks, mais au fil des mariages avec la population nubienne, ils finirent par constituer une aristocratie locale sous la suzeraineté plus théorique que réelle du pacha d'Égypte. Le territoire sous domination ottomane s'étendait jusqu'aux abords de la troisième cataracte, en amont de la ville fortifiée de Hannek. En effet, lors de leur progression vers le sud, les soldats turcs s'étaient heurtés aux troupes du sultanat fung de Sennar, qui depuis quelques décennies s'implantaient en Nubie en venant du sud. Une bataille fut livrée à Hannek entre les deux camps, à l'avantage des Ottomans. Toutefois, la conquête ne fut pas poursuivie vers le sud, la Sublime Porte se contentant d'établir entre le sud de l'Égypte et la troisième cataracte une province (turc eyalet) dont la capitale était Qasr Ibrim et qui dura jusqu'à l'invasion de l'Égypte par Napoléon. Pendant près d'un siècle, la menace fung obligea les Ottomans à maintenir une importante garnison sur l'île de Saï, portée jusqu'à 800 hommes. Mais, à partir de 1660, la révolte contre le

des origines

à la chute du sultanat Fung

\section{Les Fungs: avènement des "Sultans noirs"}

Au début du $\mathrm{XvI}^{\mathrm{e}}$ siècle apparaît au Soudan central un peuple inconnu jusqu'alors, les Fungs. Nul ne sait d'où ils viennent. Selon leurs traditions, la dynastie descendrait des fils de Marwan II, les derniers Omeyyades pourchassés par les Abbassides qui avaient cherché à se réfugier en Nubie sept siècles plus tôt (voir chapitre précédent, p.393 sq.). Mais cette légende n'est qu'une des nombreuses généalogies mythiques par lesquelles la plupart des tribus du Soudan essaient de se rattacher à des ancêtres prestigieux. On a plus sérieusement proposé qu'ils descendaient de Shillouks, le plus septentrional des peuples nilotiques, émigrés sur le Nil Bleu ou qu'ils aient leur origine parmi les tribus du Nil Bleu, non loin de la frontière éthiopienne. Il est également possible qu'ils n'aient pas initialement représenté une ethnie particulière mais une caste militaire de «janissaires noirs" constituée par les monarques d'Alodia au fil de leurs raids esclavagistes dans les marches du royaume. Leur ascension subite s'expliquerait alors par leur expérience 


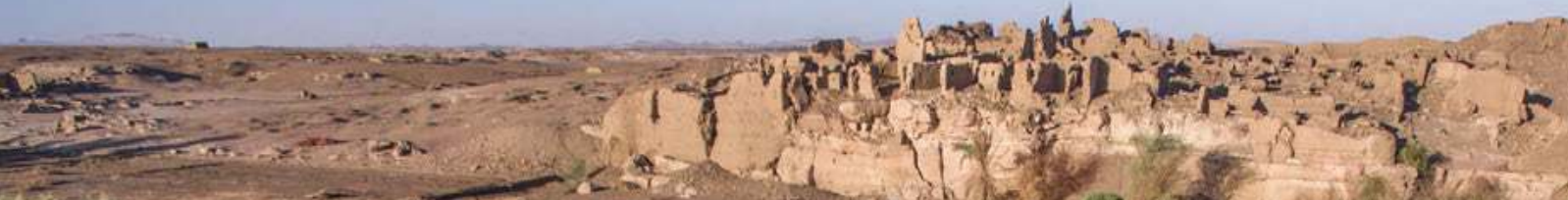

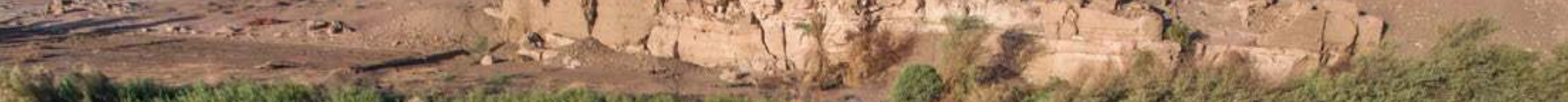

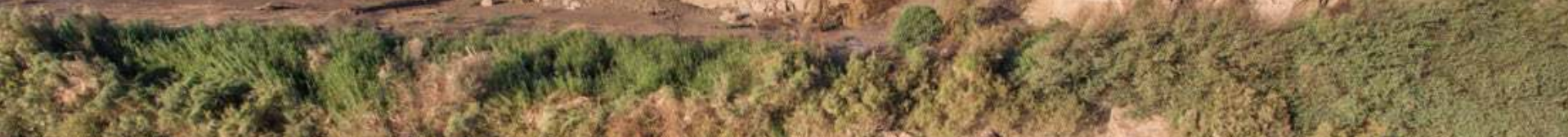

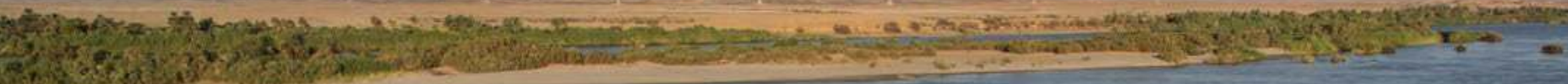

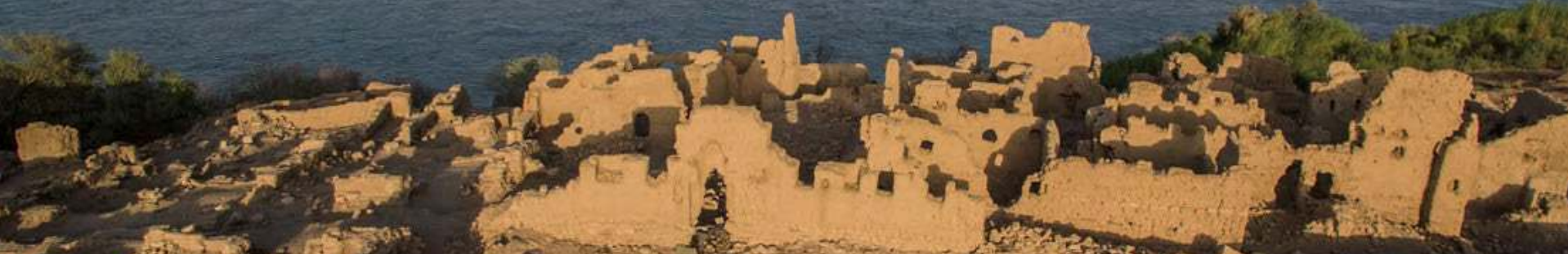
t. $x^{2}-3-2-2-1$

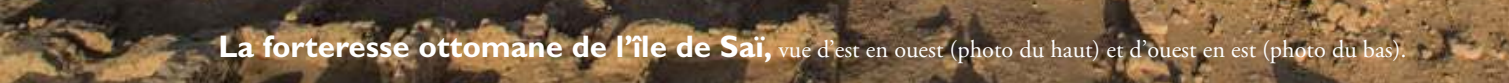

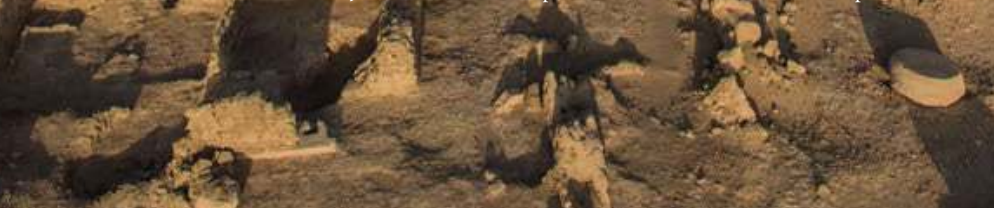




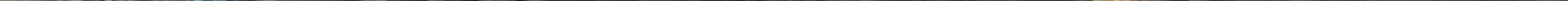


de la guerre. Les premiers califes fatimides d'Égypte avaient semblablement mis sur pied des régiments d'esclaves africains, obtenus par le Baqt ou le négoce, appelés al-Sudan («les Noirs»), dont la révolte au début du $\mathrm{XI}^{\mathrm{e}}$ siècle ne fut que difficilement écrasée.

Le premier de ces monarques fungs que l'on surnomma Sultana al-zerga, "les Sultans noirs», était Amara (ou Omara) Dunqas. Il aurait accédé au pouvoir en l'an 910 de l'Hégire, soit en 1504-1505, selon la Chronique fung. C'est lui qui aurait fondé sur le Nil Bleu la cité de Sennar, destinée à devenir la capitale du sultanat que l'on appelle aussi le «Royaume de Sennar». Toutefois, ce n'est que plus d'un siècle plus tard qu'y fut construite une mosquée, ce qui laisse supposer que cette ville n'a pas immédiatement acquis son statut de résidence royale. La première capitale fut Lamul, un site en amont sur le Nil Bleu qui n’a pas encore été localisé, et non Soba, comme le relate la Chronique fung.

Sur le premier sultan Amara Dunqas, nous possédons un témoignage direct. Un aventurier excentrique, peut-être juif oriental, nommé David Reubéni, a laissé un manuscrit en hébreu de ses nombreux voyages, y compris un séjour dans le sultanat fung où il se fit passer pour un musulman, descendant du Prophète, auprès du sultan. Il décrit ce dernier comme un homme aimable et généreux, mais il est évident que le souverain et sa cour n'avaient de l'islam qu'une connaissance très rudimentaire. Il est probable qu'ils n'aient été à cette époque que récemment et partiellement convertis. Par la suite, les Fungs feront tout pour attirer et fixer dans leur royaume des oulémas itinérants, afin de parfaire leur éducation musulmane.

des origines

à la chute

du sultanat

Fung

La chute du royaume d'Alodia et la prise de Soba, sa capitale, ne sont pas dues aux seuls Fungs. Quelques décennies avant l'avènement d'Amara Dunqas, la ville d'Arbaji fut fondée sur le Nil Bleu par une tribu arabe, à un endroit qui était précédemment au cœur du royaume d'Alodia. Peu de temps après, les tribus arabes voisines furent fédérées sous le commandement d'un certain Abdallah Jamma ("Abdallah le Rassembleur»). Cette confédération, appelée les "Abdallabs» d'après le nom de son chef, est selon la tradition responsable de la prise de Soba. Assez rapidement, les Abdallab et les Fungs devinrent rivaux pour récupérer les territoires autrefois tenus par les Alodiens. Au terme d'une bataille près d'Arbaji, les Abdallabs vaincus durent se soumettre à Amara Dunqas, qui apaisa la situation en nommant Abdallah Jamma gouverneur de la région de Qerri, au nord de l'actuel Khartoum.

La Chronique fung fut compilée et rédigée vers 1838 par un administrateur de la période turque (Turkiyya), Cheikh Ahmed. Elle reprend des archives anciennes mais n'est véritablement détaillée que pour le dernier siècle 


\section{Début de la Chronique fung}

Cheikh Ahmed ibn el-Hajj Abou Ali, dit Kateb al-Shuna, XIx siècle, d'après la traduction anglaise de G. Vantini, Oriental Sources concerning Nubia, Heidelberg-Varsovie, 1975

[I] Voici l'histoire des terres des Noubas, rapportant qui les gouverna, en commençant par les rois des Fungs, ce qui arriva durant leurs règnes jusqu'aux temps présents, qui leur succéda et comment leur royaume tomba; mais Dieu le Tout-Puissant connaît et juge mieux ce qui est caché. On raconte dans les histoires que j'ai lues que le premier chef des Fungs à être investi du pouvoir royal était le roi Omara Dunqas, qui fonda la cité de Sennar en l'an 910 de l'Hégire.

Avant cette date, les Fungs avaient vaincu les Noubas (Alodiens) et fait de la cité de Soba leur capitale. Dans cette ville, il y avait de beaux édifices et jardins et un bâtiment où résidaient les musulmans. Elle était située à l'est du Nil, non loin de la confluence de cette rivière avec le Nil Blanc. La nourriture principale de ses habitants était le sorgho blanc appelé al-qassabi. Leur religion était le christianisme et ils avaient un évêque nommé par le patriarche d'Alexandrie, comme avant eux les Noubas (comprendre "les Makourites»). Leurs livres étaient en grec mais ils les commentaient dans leur propre langue.

[II] Ces peuples furent vaincus au $\mathrm{IX}^{\mathrm{e}}$ siècle (de l'Hégire, soit notre $\mathrm{XVI}^{\mathrm{e}}$ siècle). À cette époque, il n'y avait pas d'école pour les musulmans qui vivaient parmi eux, ni respect de la loi islamique, si bien qu'une femme, dit-on, pouvait être répudiée par son époux et mariée par un autre homme le même jour sans délai de purification. Cette situation continua jusqu’à l'arrivée parmi eux de Mahmoud el-Araki depuis l'Égypte. Il leur enseigna les rudiments de la loi islamique (...).

[IV] L'islam pénétra au pays des Noubas durant le califat d'Haroun el-Rachid l'Abbasside, mais, comme nous l'avons précisé, il n’y avait pas de véritable respect de la loi.

[V] Le début du règne d'Omara Dunqas se passa au commencement de... (lacune). Le peuple se rassembla autour de lui et ne cessa de lui rendre visite alors qu'il résidait au Gébel Moya, qui est à l'est de Sennar. Enfin vint à lui Abdallah Jamma de la tribu des Arabes Kawasma, père de Cheikh Ajib al-Kafuta, ancêtre des Awlad Ajib. Ensemble, ils décidèrent de faire la guerre aux Anags, aux rois de Soba et de Gerri. 
[VI] Ainsi Omara et Abdallah Jamma et leurs troupes partirent faire la guerre aux rois de Soba et de Gerri, les vainquirent et les tuèrent.

[VII] Alors leurs peuples acceptèrent qu'Omara fût roi à la place du roi d'Alwa (Alodia), c'est-à-dire Soba, parce qu'il était le plus puissant, et qu'Abdallah Jamma prît la place du roi de Gerri.

[VIII] Ainsi, il partit fonder la ville de Gerri qui est près du Gébel el-Royyan sur la rive orientale et en fit le siège de son royaume. Semblablement, Omara fonda la ville de Sennar [d'après le nom d'] une femme appelée Sennar qui vivait là, et il en fit sa capitale. Cela se passa en l'an 910 de l'Hégire (15041505 de notre ère).

[IX] Désormais Omara et Abdallah vécurent comme des frères, mais Omara avait préséance sur Abdallah s'ils se trouvaient ensemble en un même lieu. Toutefois, si Omara était absent, Abdallah était investi des mêmes pouvoirs que ceux dont Omara était investi, et ce système demeura en place parmi leurs descendants jusqu’à la fin de leurs règnes.

le Soudan

\section{0}

des origines

à la chute

du sultanat

Fung
[X] Après la victoire des Fungs sur les Noubas, ces derniers se dispersèrent et fuirent vers le Fazugli et le Kordofan, à l'exception de quelques-uns qui s'étaient convertis à l'islam et mêlés avec les Arabes installés dans leur pays. Ils sont à présent peu nombreux et vivent dans les environs de Shendi et de Gereif Oumr. Peu de gens savent que ces hommes sont d'origine nouba, car leur langue est devenue l'arabe et leur apparence physique s'est rapprochée de celle des Arabes par suite de leur mélange avec eux. 


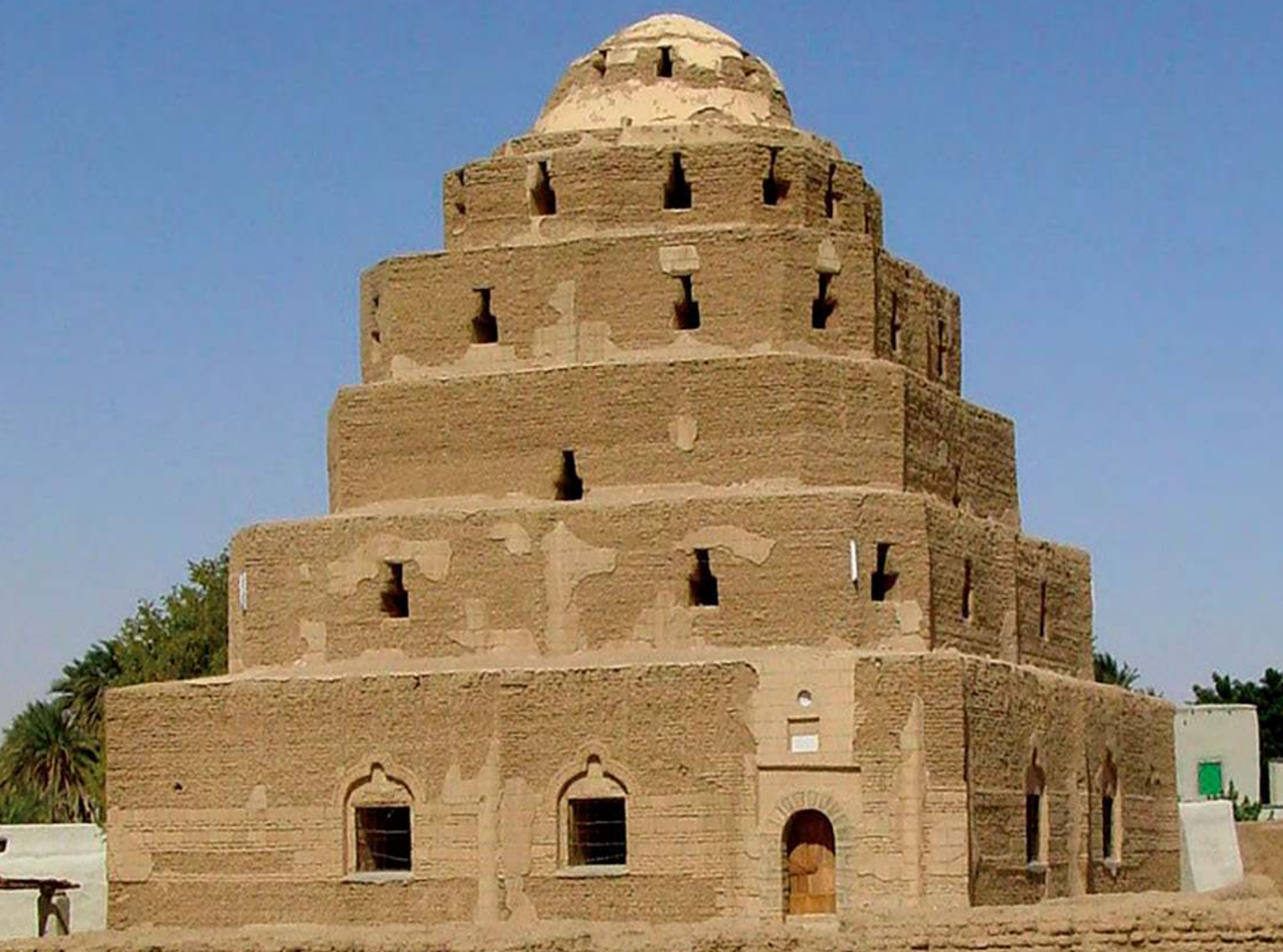


du sultanat. Nous en donnons ici les premières sections, qui racontent les commencements de la dynastie fung, son alliance avec Abdallah Jamma et la fin d'Alodia.

\section{Extension territoriale des Fungs et conflits avec leurs voisins}

le Soudan

432

des origines

à la chute

du sultanat

Fung

Durant les règnes suivants, le sultanat fung gagna les territoires autrefois tenus par les rois chrétiens de Nubie. Sous le règne d'Abd el-Gadir I ${ }^{\text {er }}$ (15511558), fils d'Amara Dunqas, les troupes atteignirent la Moyenne-Nubie où elles entrèrent en conflit avec les Ottomans, comme nous l'avons vu précédemment. À la même époque, les régions encore indépendantes situées entre le Nil Bleu et le Nil Blanc, le Gébel Moya et le Gébel Saqadi, furent annexées par le sultan. Vers le sud et le sud-est, les Fungs étaient en concurrence avec l'Empire éthiopien. Lors d'une révolte, le sultan Abd el-Gadir II (1604-1606) fut destitué et gagna l'Abyssinie. Il avait accepté de reconnaitre la suzeraineté de l'empereur Sousnéyos s'il parvenait à remonter sur le trône, ce qui ne se produisit pas. Son remplaçant, Adlan (1606-1611), régna assez peu longtemps mais parvint à écraser une rébellion de leurs anciens alliés, les Abdallabs, rois de Gerri. Le pouvoir des sultans fungs, qui devinrent alors seuls maitres de leur territoire, s'en trouva renforcé.

Aussi, lorsque le sultan Badi Ier (1611-1616), fils d'Abd el-Gadir II, accéda au trône qu'avait perdu son père, il fut extrêmement humilié de recevoir de Sousnéyos un bracelet d'or et une chaise dorée, des présents que les empereurs d'Éthiopie offraient traditionnellement à leurs vassaux. C'était un double affront, remettant en question l'indépendance du sultanat et rappelant au nouveau souverain le destin peu glorieux de son père. En guise de réponse, Badi fit parvenir à Sousnéyos une paire de vieux chevaux aveugles. C'était un casus belli, que saisit l'Empereur. Une guerre s'engagea entre les deux royaumes sous le règne de Rubat (16161645), fils et successeur de Badi I ${ }^{e r}$, lequel était mort peu après cet échange de présents insultants. Toutefois ce conflit, entre 1618 et 1619, consista plutôt en une succession d'escarmouches. Lors de la seule bataille rangée, les Éthiopiens eurent le dessus. Mais il n'y eut pas de lendemain car Sousnéyos concentra ensuite ses efforts sur l'adoption d'une réforme religieuse visant à remplacer la doctrine copte par le catholicisme, une tentative qui aboutira à la guerre civile en Éthiopie et à l'abdication de l'empereur une décennie plus tard.

Sous le règne de Badi II (1645-1680), fils de Rubat, une expédition fut lancée à l'ouest, dans le Kordofan, contre le royaume de Tégali. Cette petite principauté avait été fondée vers 1530 par un prêcheur itinérant, originaire de la province de Shendi, qui avait converti la région à l'islam. 
Elle se situait à l'est des Monts Nouba, près de la ville de Rashad. Les rois de Tégali avaient attiré des hommes de foi et des commerçants (souvent les deux à la fois) de tout le Soudan en leur distribuant des terres. Le royaume était devenu prospère et sa réputation amena le sultan fung Rubat à céder en mariage l'une de ses filles au souverain de Tégali, Geili Abou Gouroun. Mais sous le règne de Badi II, un incident se produisit à Tégali : un ami du sultan de Sennar fut molesté et dépouillé de ses biens. Geili Abou Gouroun, au cours de la dispute, avait mis au défi Badi II, son beau-frère, de venir l'affronter à Tégali. Le sultan le prit au mot et partit avec ses troupes pour le Kordofan. Toutefois, la conduite chevaleresque de Geili Abou Gouroun lors du siège de la cité toucha le cœur de Badi II. Le roi de Tégali en fut quitte pour la reconnaissance de la suzeraineté de Sennar, assortie d'un tribut annuel. Néanmoins, le sultan revint à la capitale avec un large butin d'esclaves capturés dans les Monts Nouba, qu'il installa par quartiers autour de la cité pour en assurer la défense.

Badi II s'empara également, le long du Nil Blanc, de terres occupées par les Shillouks, une importante tribu nilotique, étendant ainsi le territoire de Sennar vers l'ouest et facilitant la jonction avec le Kordofan. La gloire du sultanat durant le règne fut toutefois ternie par la révolte des Shaigiyyas, que nous avons évoquée plus haut. Elle détacha en effet du royaume fung une large portion de la vallée du Nil, la partie centrale de la grande boucle, rendant difficiles les communications entre la province septentrionale du royaume et la capitale, et dangereux le commerce caravanier entre l'Égypte et la région de Sennar. L'indépendance des Shaigiyyas ne devait cesser qu'avec la conquête du Soudan par les armées de Méhémet Ali en 1819-1821, et encore eurent-elles plus de mal à venir à bout de leur résistance qu'à s'emparer du reste du pays.

\section{Le sultanat du Darfour}

Comme nous l'avons indiqué dans les chapitres précédents, on ne possède pas de données historiques sur l'ouest du Soudan, Darfour et Kordofan, avant l'époque moderne, si l'on excepte quelques pauvres lignes chez les chroniqueurs arabes. Les récits des voyageurs occidentaux, notamment Gustav Nachtigal qui séjourna au Darfour en 1874, rapportent des traditions qui permettent de remonter quelques siècles en arrière mais ne sont pas toujours fiables. On devine des bribes du passé de ces régions par les rares et difficiles fouilles archéologiques qui y ont été menées. Ainsi, les excavations dirigées par Brigitte Gratien au début des années 2000 sur les sites de Zankor et Abou Sufyan, à la lisière entre Darfour et Kordofan, dans le haut bassin du Wadi el-Melik, ont montré 
le Soudan

\section{4}

des origines

à la chute

du sultanat

Fung que, depuis le début de l'ère chrétienne jusqu'au XIve siècle, une population y était installée dans de véritables cités fortifiées. Malheureusement, faute d'écrits, faute d'objets d'importation et de parallèles pour la céramique singulière trouvée sur le site, il n'est pas possible pour l'heure de reconstituer, même partiellement, l'histoire de ces cités. Les fouilles rapides et superficielles dirigées dans les années 1930 par le Britannique Arkell sur les sites royaux d'Ain Farrah et Turra, au nord du Gébel Marra, n'ont guère apporté d'éléments exploitables, au point qu'Arkell, qui voyait dans certains bâtiments une influence de l'architecture islamique du sultanat du Bornou, au Tchad, dut réviser son interprétation lorsqu'il eut en mains de la céramique chrétienne ramassée sur le site d'Ain Farrah quelques années auparavant.

Vers le XIII ${ }^{\mathrm{e}}$ siècle, le Darfour était divisé entre plusieurs royaumes païens dont les principaux étaient au nord celui des Zaghawas et au sud celui des Dadjos (ou Dajus). Les Zaghawas, un peuple nomade de langue nilo-saharienne, étaient très nombreux et très anciennement installés dans la région, tant au Tchad qu'au Soudan. Leur territoire, plus étendu encore auparavant, fut amputé de larges portions à l'ouest par son voisin le sultanat du Kanem. Mais c'est surtout la migration de nombreuses tribus arabes depuis l'Égypte à partir de la fin du XIv e siècle qui mit fin au royaume zaghawa. Le royaume dadjo était centré sur la région du Gébel Marra, au sud du Darfour. On ignore à quelle date il fut fondé. Les Dadjos sont une ethnie de langue nilo-saharienne, aujourd'hui dispersée depuis le Tchad jusqu'à l'est des Monts Nouba, au Kordofan.

À une époque indéterminée, mais sans doute avant la fin du $\mathrm{XIV}^{\mathrm{e}}$ siècle, les souverains dadjos furent vaincus par un nouveau peuple, les Toungours (ou Tounjours), qui s'empara également du royaume voisin du Ouaddaï, dans l'est du Tchad. Les Toungours sont aujourd'hui musulmans et arabophones et font remonter leur origine à Tunis. Mais il semble bien qu'ils provenaient plutôt de Dongola, comme le rapportent des traditions alternatives, et le nom nubien de la capitale de Makouria, Tungul, est une bien meilleure étymologie pour leur nom que Tunis. Le premier roi toungour du Darfour aurait été Ahmed Ma'agur, "Ahmed aux jarrets coupés», dont le second élément semble une déformation du nom de Makouria, alors que le premier est une réfection anachronique, l'islam n'étant apparu que plus tard dans la région.

De très nombreux emprunts au nubien du Nil dans les langues de la région montrent sans ambiguïté la présence à un moment donné d'une importante entité politique de langue nubienne, qui ne peut être autre que le royaume toungour. La découverte de céramiques à motifs chrétiens dans ce qui semble les ruines d'un monastère à Ain Farrah, siège du dernier roi 
toungour du Darfour, Shaw Dorshid, montre que le christianisme était la religion de l'élite, même si les traditions orales ultérieures ont occulté ce point pour d'évidentes raisons politiques. Les sultans du Darfour, par la suite, ont en effet revendiqué l'héritage des Toungours tout en se présentant comme les champions de l'islam.

Vers le milieu du XVI ${ }^{\mathrm{e}}$ siècle, les Toungours du Darfour furent renversés par une nouvelle dynastie, les Keira, issus de la principale ethnie de la région, les Four, auxquels elle doit son nom actuel, Dar-Fur ou "pays des Four». Le premier sultan keira, Suleiman Solong, est réputé avoir introduit l'islam dans le royaume, d'où peut-être son surnom de Solong ou Solongdungo, «l'Arabe» en langue four. Sous son règne, une partie du clan keira se révolta. Battus militairement, ils s'exilèrent dans le Kordofan où ils créèrent sous le nom de «Musaba'at» un sultanat indépendant. Vers 1670, le fils de Suleiman Solong, le sultan Musa, lui succéda dans le palais de Turra. Mais après son règne, chacun des souverains se fit construire un nouveau palais (en arabe local fasher) en un nouvel endroit, jusqu'à ce que le sultan Abdelrahman al-Rachid, à la fin du XviII ${ }^{\mathrm{e}}$ siècle, choisisse un site, près du lac Tendelti, qui resta jusqu’à nos jours la capitale du Darfour, à laquelle on donna le nom d'el-Fasher, «le Palais».

Sous le règne du fils de Musa, le sultan Ahmed Bukr (env. 16821730), le royaume s'étendit, ce qui mena à des conflits avec ses voisins, notamment le sultanat du Ouaddaï où, vers 1635, les Toungours locaux avaient également été renversés par un chef arabe qui y établit l'islam. Vainqueur du Ouaddaï, Ahmed Bukr mit sur pied une armée bien entrấnée qu'il équipa d'armes à feu grâce au commerce avec l'Égypte à laquelle le sultanat était relié par une route caravanière célèbre, la Piste des Quarante jours (darb el-arba in). Comme les sultans fungs, il favorisa l'installation de docteurs de la loi venus de l'étranger pour répandre l'islam, qui jusqu'alors était confiné à la sphère dirigeante.

Ses fils, Mohamed Dowra, Omar Leel, Abulgasim et Mohamed Teirab, qui lui succèdent l'un après l'autre sur le trône de 1730 à 1787, furent moins avisés. Les deux premiers se firent remarquer par leur cruauté. Les suivants se lancèrent dans des campagnes hasardeuses (le premier contre le sultanat du Ouaddaï au Tchad, le second contre le sultanat rival des Musabaáa au Kordofan) et ils y perdirent la vie. Toutefois, une bonne partie du Kordofan fut ainsi annexée par le successeur de Mohamed Teirab, le sultan Abdelrahman al-Rachid (1787-18o2), et resta sous le contrôle du sultanat keira jusqu'à l'arrivée des troupes de Méhémet Ali en 1821. Le Darfour lui-même échappa à l'annexion et, à part une période de rattachement au Soudan entre 1874 et 1899, resta indépendant jusqu'en 1916, quand les Anglais vainquirent et tuèrent le dernier sultan, Ali Dinar. 


\section{Le dernier siècle du sultanat fung}

À partir de la fin du Xvir e siècle, l'histoire du royaume de Sennar n'est qu'une longue décadence où se succèdent les guerres civiles et les coups d'État. Badi III (1692-1716), dit "le Rouge», s'aliéna une partie de la population et dut mater la révolte conduite par un prétendant au trône. Son fils Ounsa III fut déposé au bout d'un an de règne en raison de ses mœurs dissolues et remplacé par un membre d'une lignée indirecte, le sultan Noul, qui mourut au bout de quatre ans. Son fils et successeur Badi IV, malgré un long règne (1723-1761), n'eut à son actif qu'une victoire contre une tentative d'invasion éthiopienne en 1744. Mais il ne put la repousser que grâce à l'aide d'une faction des Musaba át, le sultanat four dissident du Kordofan (voir section précédente), qui avaient été intégrés dans l'armée fung sous la direction de leur chef Khamis ibn Jangal. Ce dernier écrasa les troupes de l'empereur abyssin Jésus II sur la Dinder. Les initiatives personnelles de Badi IV furent plus malheureuses. Il engagea une série de réformes impopulaires, qui visaient notamment à placer à des postes importants des hommes de confiance choisis parmi les descendants d'esclaves des Monts Nouba, au détriment des représentants de la vieille noblesse fung. Un des généraux que Badi IV avait placé à la tête des possessions du Kordofan suite à ses victoires contre l'ennemi, Mohamed Abou al-Kaylak, prit la tête des nobles mécontents et, suivi de son armée, s'avança vers Sennar où il déposa le sultan.

Les aristocrates fungs avaient sans le savoir fait le pire des choix. Abou al-Kaylak n'était pas fung lui-même, mais hamag, une ethnie d'origine obscure qui représente peut-être ce qui restait de la composante nubienne du royaume de Sennar. Il agit dès lors pour son compte et celui de sa tribu, faisant et défaisant les rois à son gré et n'hésitant pas à supplicier les nobles fungs qui s'opposaient à lui. Il plaça le fils de Badi IV, Nasser, sur le trône, puis le déposa huit ans plus tard et le fit mettre à mort quand il voulut se révolter. Abou al-Kaylak choisit un nouveau sultan en la personne d'Ismaïl, frère du précédent.

Nous possédons du royaume de Sennar sous le règne d'Ismaïl et le gouvernement du régent Abou al-Kaylak un témoignage très précis, celui de James Bruce. Cet aristocrate écossais effectua entre 1768 et 1773 un grand voyage à travers l'Afrique orientale, dont le but principal était de découvrir les sources du Nil. Il passa notamment deux ans auprès de la cour d'Éthiopie, avant de visiter près du Lac Tana les sources du Nil Bleu, qu'il considéra comme le fleuve principal. Alors qu'il revenait vers l'Égypte, il fut détenu quelque temps dans le royaume de Sennar en 1772, avant de pouvoir continuer vers l'Égypte. Le récit de cette expédition fut publié assez tardivement en 1790. 


\section{Description du royaume fung de Sennar}

James Bruce, Travels to Discover the Sources of the Nile, 1790, Tome Iv, 479-481

Il y a trois principaux gouvernorats dans le royaume de Sennar. Le premier est à el-Ais (Kowwa), la capitale du pays dont viennent les Shillouks. Le Bahr el-Abiad (Nil Blanc) se répand sur tout le territoire et, divisé en une multitude de petits chenaux — naturels ou artificiels, je l'ignore entoure un grand nombre de petites îles, sur chacune desquelles s'élève un village, et cet ensemble de villages est appelé el-Ais. Les habitants sont tous pêcheurs et possèdent nombre de bateaux ressemblant à des pirogues, avec lesquels ils descendent et remontent les cataractes. C'est avec une incroyable flottille de ces bateaux qu'ils envahirent la région lorsqu'ils entreprirent sa conquête contre les Arabes, les prenant complètement au dépourvu. Ils n'avaient à cette époque aucune arme en fer, leurs épées et leurs lances étant faits d'un bois dur appelé dengi-sibber. Le gouverneur d'el-Ais doit être un parent du mek (roi) de Sennar et n'est jamais autorisé à quitter son poste ou à se rendre à Sennar.

Le deuxième gouvernorat, second en importance après le précédent, est le Kordofan. Son tribut principal consiste en esclaves venus de Dyre (Gébel el-Dair) et Tegla (Tégali). Il semble que ces endroits soient les plus commodes pour envahir ces montagnes, soit qu'ils disposent de points d'eau sur la route, soit pour une autre raison inconnue. Mahomet Abou Kalec (Mohamed Abou al-Kaylak, régent du royaume fung) était à la tête de ce gouvernorat avec mille chevaux noirs équipés de cottes de mailles, ce qui lui permit de rester alors indépendant du roi. C'est une région frontalière avec le Darfour, un État noir encore plus barbare, s'il est possible, que Sennar. Le Darfour l'a souvent conquise et reconquise sur Sennar.

Le troisième gouvernorat est Fazuclo (Fazugli), bordé par la rivière el-Ais [Nil Blanc] à l'ouest, le Nil [Bleu] à l'est et au sud, par les montagnes de Fazuclo, où il $\mathrm{y}$ a de grandes cataractes. Ces sommets font partie de la large chaîne montagneuse de Dyre (Gébel el-Dair) et Tegla (Tégali), qui se poursuit vers l'est jusqu'à cette partie du continent et dont provient la majeure partie de l'or et des esclaves qui font la richesse de ce pays. L'or constitue en effet le tribut principal de Fazuclo. Le gouverneur n'est pas un Fung, mais précisément le prince contre lequel l'armée de Sennar conquit le pays. C'est un point de civilisation remarquable dans cette nation barbare et une politique qui a dû être couronnée de succès, puisqu'ils l'appliquent constamment, que de faire du prince de l'État qu'ils ont conquis leur lieutenant dans le gouvernement ultérieur de son propre 
pays. Tel avait été le cas avec Dongola, dont ils laissèrent en place le mek (chef), mais aussi avec Wed Ageeb, prince des Arabes (Ajib al-Kafuta, chef des Abdallabs, second roi de Geiri). Ce fut aussi le cas dans le Fazuclo, le Wed Aboud, le Jibbel Moia (Gébel Moya) et bien d'autres petits États qu’ils conquirent tous sans en changer le prince.

Les troupes à Sennar, tout autour de la capitale, sont constituées d'environ 14000 Noubas (ressortissants des Monts Nouba) qui combattent nus, sans autres armes qu'une courte javeline et un bouclier rond — d'assez mauvais soldats, je suppose; de 1800 chevaux, tous noirs, montés par des esclaves noirs équipés de cottes de mailles et sans autre arme qu'une large épée slavonne. Je crois que, par le poids et la puissance de l'homme et du cheval, ils pourraient abattre ou disperser n'importe quelle troupe au monde, même deux fois plus nombreuse. Nul, sans avoir vu cette cavalerie, ne peut avoir la moindre idée de la perfection à laquelle atteint ici le cheval. Le mek (roi) n'a pas un seul mousquet dans toute son armée. Outre cette cavalerie, il y a un nombre indéfini d'Arabes qui paient leur tribut directement au mek et aux hautes autorités du gouvernement et vivent sous leur protection à proximité de la ville. Ils ont ainsi l'avantage de commercer avec elle, de l'approvisionner et doivent sans doute contribuer à l'armer et la défendre en cas de besoin.

\section{8}

des origines

à la chute

du sultanat

Fung

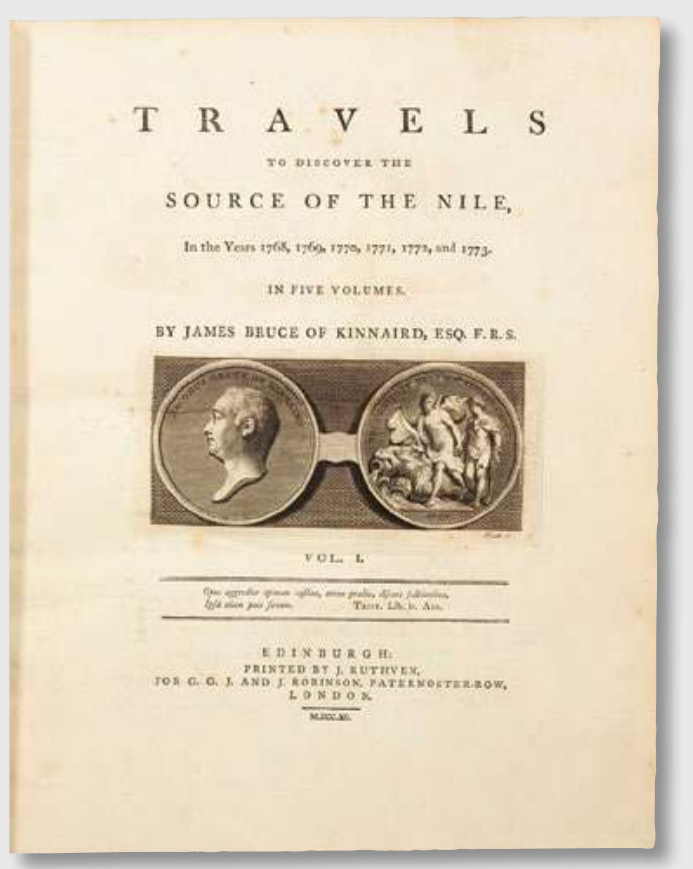

James Bruce,

Travels to Discover the Sources

of the Nile, Londres,

1790, page de grand titre

du premier volume. 
En 1777, le régent Mohamed Abou al-Kaylak mourut, mais son fils Badi wad Rajab lui succéda. Il exila le sultan Ismaïl, qui avait comploté contre lui, et installa sur le trône de Sennar le frère du roi déchu, Adlan II. La lutte entre les sultans et les régents, avec son lot de conspirations, de batailles, d'exils et d'assassinats, continua jusqu'à l'arrivée des troupes de Méhémet Ali en 1820-1821.

C'est un royaume en totale décomposition qu'attaquèrent les troupes turco-égyptiennes, avec un succès prévisible. Le dernier régent venait d'être assassiné et le dernier sultan de Sennar, Badi VII, offrit la reddition de la capitale sans grand état d'âme. Ce dernier acte mettait fin à vingt-cinq siècles d'indépendance du Soudan. Le pays avait réussi à résister aux Perses, aux Macédoniens, aux Romains, aux Arabes. Il s'était nourri des cultures de la Méditerranée sans se soumettre à leurs Empires. Il avait changé trois fois de religion sans pour autant s'assujettir à un joug étranger. La domination turque serait toutefois de courte durée. Soixante ans plus tard, avec la révolte du Mahdi, le Soudan allait connaître une nouvelle période d'indépendance

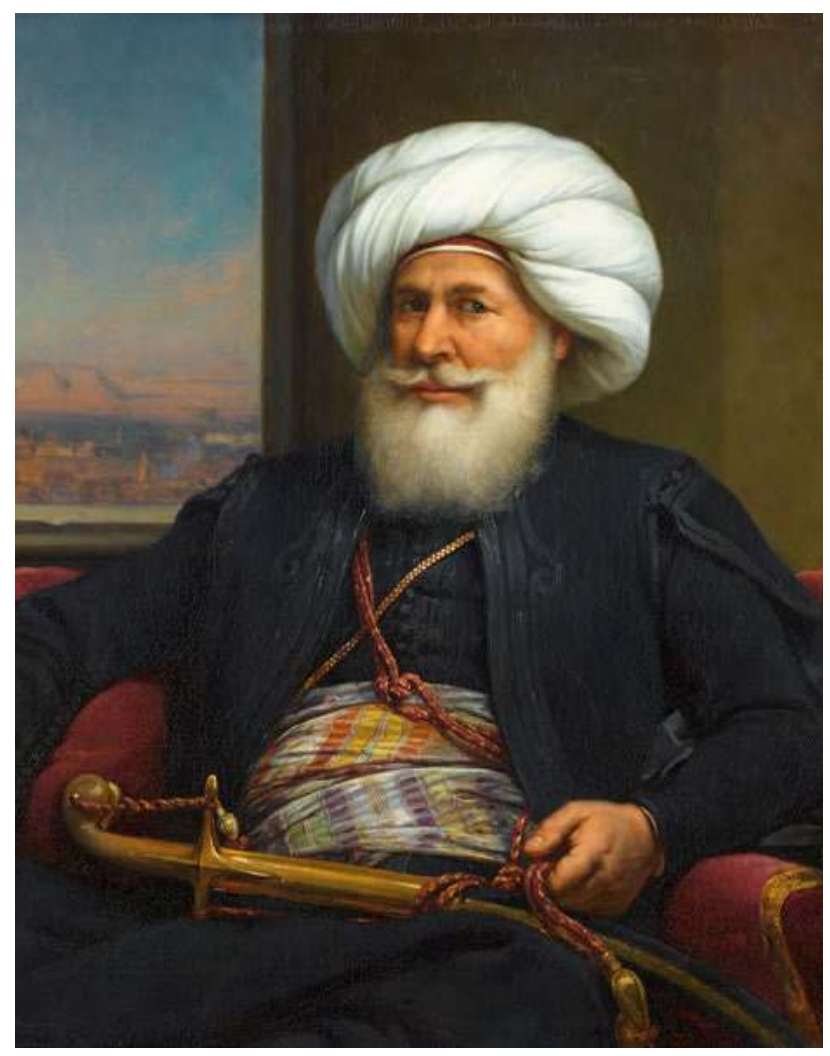

